\title{
Application and Research of Health Management System Based on Mobile Client
}

\author{
Qian Yao \\ Health Management Department, Xi'an Medical University, Xi'an, 710021, China
}

Keywords: health management; system; mobile client; data visualization; health information

\begin{abstract}
: in accordance with incomplete statistics, there are 320 million of patients with chronic disease in China. It has become a large hidden danger threatening China's national health. Therefore, to provide effective health intervention, prevention and treatment means for patients with chronic disease and high risk group, health management monitoring system based on intelligent mobile terminal is researched and developed in medical field with the help of mobile communication technology with rapid development. This system provides accurate and efficient health management services anytime and anywhere for users with chronic disease. It finally helps users establish favorable living habits and achieve intelligent management of individual health recourses through daily real-time health reminding and service recommendation. This paper focuses on application of such mobile health management system for discussions.

This paper studies application and design of health management system based on mobile client. The birth of such system represents the progress of China's medical science and mobile information field. Their objective is very clear, i.e. make people know changes of their health indicators anytime and anywhere without going out. This also includes sending considerate and humanized medical services to monitoring objects from medical center, such as pathology trend analysis graph and real-time experience report of monitoring objects.
\end{abstract}

\section{Health management}

(I) Overview

Health management refers to the whole process of conducting health management of health conditions of individuals and groups and potential crisis factors. Its aim is to mobilize physical health monitoring enthusiasm of individuals, groups and society and improve importance of national health quality.

(II) Current situation of foreign health management

In 1950s, health management as a new type of insurance service was promoted. It appeared as American national health insurance. Until 1973, American government established Health Management Organization (HMO) and made health management legislation pass Health Maintenance Act. All these aim to control the rising medical expenditure in American society. From 1987, some private enterprises in America provided perfect health management services for their employees, i.e. "health track" (PPO). Health track system is an application program which can track blood pressure, weight and other health parameters of monitoring objects. This system utilizes color coding to express the income and loss of physical health. Blood pressure of monitoring objects is confirmed according to blood pressure regulation simulation guided by American Heart Association. Green means normal, while yellow means early warning stage of relatively high blood pressure, as shown in Fig.1. 

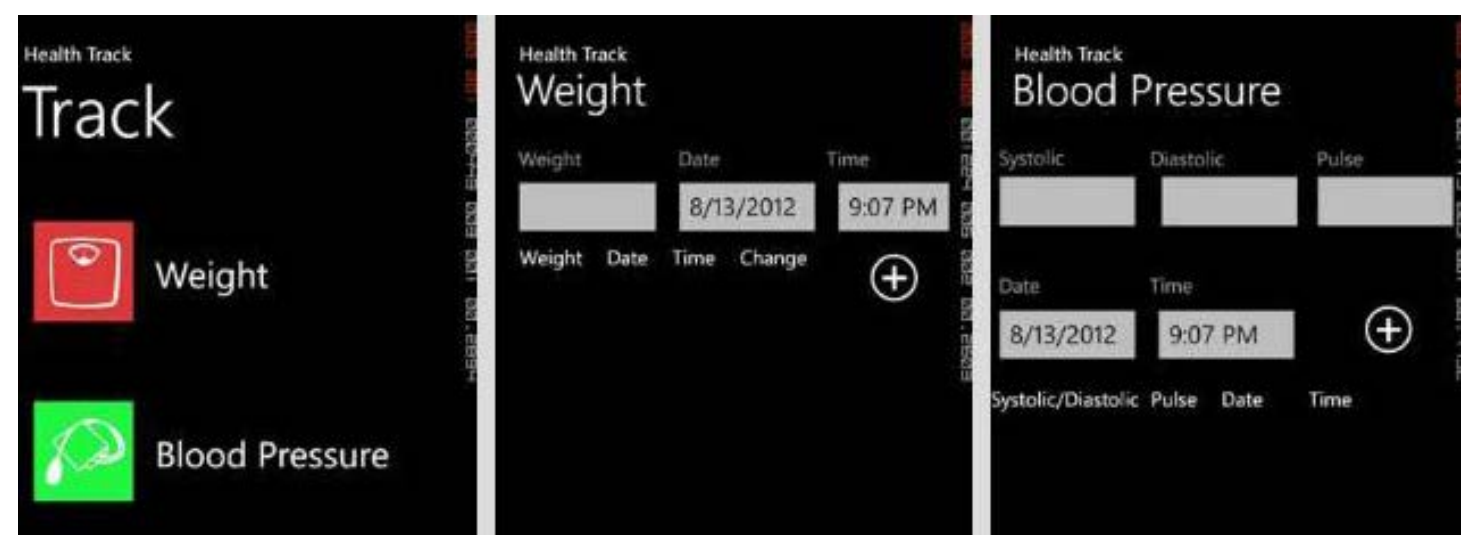

Fig.1 Schematic diagram of blood pressure of monitoring objects of health track application software

Such blood pressure monitoring application software well controls and intervenes in morbidity of more than 10 health risk factors including high blood pressure and hyperlipidemia. At present, it has covered 200000 people in America. The researches of American population health medical institution show USD 40 million of medical expenditure could be saved for the whole country annually through applying such application software to carry out long-term health management of monitoring objects. The ratio of its total cost to efficiency is 1:3.31. Till now, the people enjoying PPO program in the whole America have exceeded 100 million. This number almost reaches $60 \%$ of American population. For American people preferring to high heat in their diet, popularization of such health management application system undoubtedly becomes important contribution influencing future development of American national heath.

(III) Current situation of China's health management

Health management develops late in China. Registration authorities with health management as their basic business appeared in the end of the century. The main business of such organizations is to improve and optimize physical health of monitoring objects and provide health management services with non-drug treatment. Since SARS crisis in 2003, the state has paid more attention to the importance of health management on national health quality. Some emerging health management institutions such as "health managers" and "nutritionists" also receive more and more attention. The service objective of these employees is to give personalized and targeted health management plan as well as corresponding health recommendation in accordance with users' actual health conditions. However on the whole, China's health management services still have many defects. For example, management contents are not diversified enough; there is short of health risk evaluation; service objects are limited. But in a long run, the general direction of China's health management is still correct. As people's living standards improve continuously, their protection awareness for their own health is also enhancing continuously. So, current health management has transformed to prevention type and health care type from treatment type. In 2009, China established resident health record service. It is a national health management standard and involves everyone in China. The establishment of health records has material significance for promoting China's national health plan. It also lays a foundation for national strategic plan of "health China 2020". Over the years, health management system based on mobile client also has gradually become hot services of medical centers in each city. It can effectively and conveniently help patients know diseases, establish corresponding healthy living habits, finally gradually improving individual health stage and achieves favorable control of chronic disease through reducing potential dangerous factors of diseases.

\section{Mobile client of health management system}

According to China's national situations, although national health management based on mobile client is difficult, its significance is extraordinary. In addition, China's medical field is making efforts to this direction. Seeing from existing technology in the world, health management system 
mainly includes case query, health education, real time monitoring and other health information service functions. The system helps people establish scientific health concept through popularization of health knowledge. Through daily specific health reminding and recommendation, it helps people get rid of unhealthy living habits and prevent diseases. Service objects of client system are patients with chronic disease who should pay close attention to their health level. Such group has specific demand for system functions and properties. The specific demands are described in detail as follows:

(I) Functional demand

Whether mobile client of health management system is satisfying? Its function is in the public eye. This is also users' direct demand for the system. Current health management system will adopt multi-functional modularized management mode. In short, it carries out systematic classification of health management functions and makes entire management structure clear. The basic functions which have been realized include the following:

1. User login

Every user will gain exclusive account and password for the convenience of system login and establishing personal file. After login, intelligent terminal will save essential information of users and individuals and medical history etc. Meanwhile, the terminal will send personal information to server terminal of remote medical center for concentrated saving and analysis. In the medical center terminal, doctors can check users' state of diseases through authorization, know development and changes of various health indexes and provide effective and reliable diagnostic basis for follow-up treatment.

2. Disease query

Disease query platform aims to bring convenience for users to know disease knowledge. Query system of disease query platform is humanized. It helps users inquire relevant contents from each perspective, such as disease name retrieval, symptom manifestation retrieval and aetiological agent retrieval. Client server terminal will soon seek disease types they want from the disease database and display detailed information of diseases. This is also a service mode of "auto-diagnosis" supplied by the disease query platform for users, involving health education branch platform. Users can learn and know maintenance of physical health and disease prevention knowledge through this platform.

(II) Property demand

For users, property requirement of health management system is system operation experience demand. Some users will put forward demands for the system while using the client.

1. UI

Whether UI design is humanized is the key about whether the system can be popularized. Considering the aged occupy the overwhelming majority among users of health management system, the design of interactive interface must be concise, convenient and fast in operation. Different interface styles of the system and color design should be unified and harmonious. From visual sense, the most comfortable application experience should be provided for users.

2.Safety

At present, when network is used to transmit user information, a possible problem is information data safety. Therefore, to avoid information disclosure or missing, data encryption is adopted in management system to protect communication safety, and corresponding encryption will be conducted for personal health data.

3. System expansibility

Health information management system adopts dynamic and extensible design philosophy. During users' daily use, users' preference to system and health data will keep regular update. So, the system can maintain timely and correct response to users' current stage. Server terminal of medical center will update and back up users' individual health data so as to ensure normal operation of system and data safety. 


\section{System function design}

In China, mobile terminal in health management system gradually shows its superiority during monitoring and managing health conditions of patients with chronic disease. Most health management systems are dominated by monitoring basic physiological parameters of human body. Because there are many human parameters most chronic diseases involve, handling capacity of mobile terminal system is limited. Some characteristic data will be collected according to rules of medical science and analyzed to achieve health recommendation and various services. Meanwhile, for health data in need of continuous observation, the system will save historical data for a period and generate corresponding graphs so as to reflect development trend of state of an illness and provide necessary reference for users or attending doctors. Currently, health management system design based on mobile terminal mainly includes three parts.

(I) Health supervision

Health supervision is the core function of the whole management system. It can receive physiological data, analyze and handle data through mobile terminal. It can display objects' pulse, ECG, SPO2, heart rate as well as other physiological characteristic values and relevant waveform data. This is real-time monitoring function of health supervision. By use of mobile information technology, health supervision can be classified into three types:

1. Wireless communication of mobile monitor

At present, common monitoring communication technology in hospitals include Bluetooth, ZigBee and Wi-Fi. Zigbee can be fast to access network with low power consumption, but it is not matched with current smart-phone system standard. So, it is unsuitable for communication with users. Thus, generally hospitals will not choose ZigBee. Although Wi-Fi owns very fast transmission speed, transmission distance is very limited. Besides, the coverage is small, but the power consumption is too large. So, Wi-Fi cannot satisfy basic requirements of application anytime and anywhere. Relatively, Bluetooth in smart-phone performs well. China issued Bluetooth4.0 system in 2012 for operation and application of health management system.

\begin{tabular}{|c|c|c|c|}
\hline $\begin{array}{c}\text { Operating frequency } \\
\text { range }\end{array}$ & ZigBee & Bluetooth & Wi-Fi \\
\hline Transmission rate & $250 \mathrm{Kbps} / 40 \mathrm{Kbps} / 20 \mathrm{Kbps}$ & $1 \mathrm{Mbps}$ & $11 \mathrm{Mbps} / 54 \mathrm{Mbps}$ \\
\hline $\begin{array}{c}\text { Communication } \\
\text { distance }\end{array}$ & $10-75 \mathrm{~m}$ & $2-10 \mathrm{~m}$ & $100 \mathrm{~m}$ \\
\hline $\begin{array}{c}\text { Equipment access } \\
\text { time }\end{array}$ & $30 \mathrm{~ms}$ & $3 \mathrm{GHz} / 5.8 \mathrm{GHz}$ \\
\hline Connection time & $15 \mathrm{~ms}$ & $>3 \mathrm{~s}$ & $5 \mathrm{~s}$ \\
\hline Power consumption & Transmitting power $1 \mathrm{mw}$ & Working state $30 \mathrm{~mA}$ & $250 \mathrm{~mA}-350 \mathrm{~mA}$ \\
\hline Networking quantity & 65535 at most & 256 at most & Very large capacity \\
\hline Networking structure & Star shape, tree shape, net & Ad-hoc, scattered & Large-scale network \\
& shape & met & \\
\hline
\end{tabular}

Fig.2 Technical indexes of ZigBee, Bluetooth and Wi-Fi

Bluetooth adopts scattered network structure. Its general standard is IEEE802.15. It mainly adopts TDD (time division duplex) transmission means to achieve full duplex transmission. Android and iOS systems support networking protocol of Bluetooth. So, its application range is 
wide with strong transmission capacity.

2. Acquisition of users' physiological parameters

The major task of portable monitor is to acquire users' physiological data as the basis of users' health and illness state monitoring. What it carries is monitor and Bluetooth communication module of user's mobile terminal. Communication mode between them is asynchronous serial mode. Besides, it follows Bluetooth communication protocol and ensures data exchange stability. In health management system, basic format of data package of Bluetooth module is shown in Fig.3.

\begin{tabular}{|l|l|l|l|l|l|l|l|}
\hline $\begin{array}{l}\text { Package } \\
\text { ID }\end{array}$ & $\begin{array}{l}\text { Data head } \\
1\end{array}$ & $\ldots .$. & $\begin{array}{l}\text { Data head } \\
\mathrm{M}\end{array}$ & Data 1 & $\ldots .$. & Data N & Checksum \\
\hline
\end{tabular}

Fig.3 Basic format of data package

Data package is the key to sending data information to mobile terminal by the monitor. It mainly includes monitor self-inspection, monitoring information, system state, user information, query command and self-inspection command etc. These modules will judge data types according to different package ID. Considering some data need encryption, if users' data do not comply with verification password in unpacking process, data transmission will automatically give up or require re-transmission. This to a large extent ensures safety of data package in tge transmission process.

(I) Health data management

Personal health data refer to basic physiological parameters of human body. Usually, they can serve as specific indexes of health condition judgment and are also important basis for doctors' diagnosis. Common health data include the following:

1. Heart Rate refers to heartbeat times per minute. This is the fundamental datum in medical treatment. Especially in first-aid, Heart Rate can serve as basic basis for medical workers to rescue patients.

2. Electrocardiography is an electrophysiological activity information datum which expresses heart's activities. It can represent physiological activity curve of heart in detail.

3. SPO2 refers to percentage of hemoglobin combined with oxygen to all hemoglobin.

\section{Conclusions}

At present, most health management systems can collect, save and analyze users' physiological data in mobile terminal device, generate graphs and provide timey and accurate reminding and health recommendation for users. Meanwhile, it provides accurate and reliable basis for attending doctors to carry out follow-up diagnosis. Health management system provides convenient, fast and sustainable solutions for health resource management of individuals and the whole society under the support of mobile communication platform. Ultimately, it will become an indispensible part of wisdom medical treatment.

\section{Acknowledgments}

This paper is a part of special fund program of Shaanxi Education Department (program No.: 2013JK1153)

\section{References}

[1] Li Yaping, Design and implementation of mobile health monitoring management system [D]. Southern Medical University, 2014

[2] Zhao Yiling, Design and implementation of Android-based herdsman health management client

[D]. Beijing University of Posts and Telecommunications, 2013

[3] Zhang Zhixun, Wang Juan and He Hui, Design and implementation of remote health monitoring system [J]. Industrial Instrumentation \& Automation, 2014,01:20-22+91 